Proceeding of the International Conference on Advanced Science,
Engineering and Information Technology 2011

\title{
Projection Based Region of Interest Segmentation in Breast MRI Images
}

\author{
Sim Kok Swee ${ }^{\#}$ Chia Fu Keong ${ }^{\#}$, Chong Sze Siang ${ }^{\#}$, Tso Chih Peng ${ }^{*}$, Siti Fathimah Abbas ${ }^{\#}$, Sarimah Omar ${ }^{\#}$ \\ ${ }^{\#}$ Faculty of Engineering and Technology, Multimedia University, Malacca, Malaysia; \\ *School of MAE, Nanyang Technological University, Singapore; \\ ${ }^{\#}$ Malacca General Hospital, Malacca, Malaysia.
}

\begin{abstract}
In this paper, a computer aided design auto breast region segmentation system is presented to identify the region of interest (ROI) in magnetic resonance imaging (MRI) images. The system is proposed due to the necessary for performing useful postprocessing on the image for breast cancer research and treatment. Besides, while the ROI is segmented, the image post-processing efficiency of the system is greatly improved. The vertical and horizontal projections algorithms are employed to refine the breast ROI. The methodology has been applied on 55 sets of Digital Image and Communications in Medicine (DICOM) breast MRI datasets images. The experimental results show that the system is able to segment the breast ROI accurately.
\end{abstract}

Keywords - magnetic resonance imaging, breast region, region of interest, segmentation, vertical projection.

\section{INTRODUCTION}

Breast cancer is the unmanageable cells growth that originated from breast tissues, and most normally from the inner lining of milk ducts or the lobules to the nipples. Most types of cancer cells eventually form a lump or mass called a tumour, and are named after the part of the body where the tumour originates. According to the information from the American Cancer Society 2009, an estimated 192,370 new cases of invasive breast cancer will be diagnosed among women, as well as an estimated 62,280 additional cases of in situ breast cancer. Besides, approximately 40,170 women are expected to die from breast cancer [2]. Magnetic Resonance Imaging (MRI) plays a central role in the process of detecting abnormalities in breast cancer screening.

Mammography is one of the standard screening tools. However, mammography is less sensitive for young women with a hereditary risk [3]. When a case is undetermined by mammography, Magnetic resonance imaging (MRI) serves as an alternative imaging tool because it has higher sensitivity than mammography [4]. MRI can detect breast cancers in women at high risk which appear occult in mammography [5], hence it is useful for disease staging and therapeutic response monitoring for breast cancer patients. MRI is not replacing traditional mammography as a screening tool because of the relatively high running cost, but used as an adjunct to mammography in the surveillance of high risk populations.

In breast cancer research, MRI is being studied and explored as a prospective tool for monitoring tumor size and assessing response to contrast reagents. Recently, researchers and radiologists often interpret MRI images manually, but as MRI gains greater acceptance in the clinical realm, the need for rapid and robust automated image processing techniques is necessary. In order to process the raw images and extract the required data, it is first necessary to segment the region of interest (ROI) of the image. Basically, the ROI should include all fibroglandular (breast) and fat tissue but exclude other unwanted portions such as the skin, chest wall, and image artefacts.

With the improvement of MRI, the slices collected per patient are increased. A full set of MRI breast screenings composed of 144 slices from different positions. Since hundreds of images and scans are required for the screening exam, a considerable time must be consumed into indentifying significant ROI. Besides, more computation costs are spent while entire image is being processed 
without ROI. In this scheme, a ROI identification system is designed in order to increase the convenience of doctors and radiologists due to the automated ROI recognition. In addition, the computation cost of the insignificant portion is eliminated and post-processing efficiency is increased.

\section{Proposed Method}

The proposed scheme utilizes the idea from the vertical projection in character recognition. Character Recognition, also known as Optical Character Recognition or OCR, is concerned with the automatic conversion of scanned and digitized images of characters in running text into their corresponding symbolic forms. The humans read poor quality machine print or thousands slices of breast MRI are time consuming.

According to the approach [6] for character recognition of old Bulgarian text documents, most OCR systems have binarization as a pre-processing step. It uses vertical projection on horizontal axis on in advance inclined text characters. In this transformation the projection contour assumes different type from standing characters. By employing this approach, a MRI breast ROI segmentation system is developed successfully. The algorithm combines the vertical projection on horizontal and vertical both axes. Besides, the histogram of breast MRI is plotted and analyzed.

\section{A. Data Acquisition}

The data used in this scheme consist of 55 set MRI scans. The raw data are acquired from Malacca General Hospital, Malaysia and the biopsy results are included as reference. The images have resolution 448 x 448 pixels and are in greyscale format where pixel intensity represented by a 16 bits integer. The MR images were taken with the T1 mode, FLASH 3D traverse setting of the MRI machine. Sequences of images were generated every one minute for a total of six minutes. For other settings such as echo time (TE) and repetition time (TR), default value is used to standardize the output images.

\section{B. Image Pre-Processing}

In order to provide high accuracy result for the MRI screening tests, a series of algorithms for noise elimination are required. The patient's motion that often results from involuntary movement such as respiration, cardiac motion, and blood flow is the major physiological effect that causes the artefact and influences the precision of the screening test. The sequence of MRI images captured which show inconsistencies in phase and amplitude will lead to signal burring and false cancer detection. The motion artefact is reduced physically by using breast holding, cardiac synchronization or respiratory compensation techniques. However, in order to eliminate the motion artefact in breast MRI slices, a background subtraction technique has been employed to create an in-phase sequencing and connecting object. Figure 1 shows the motion artefact in breast MRI.
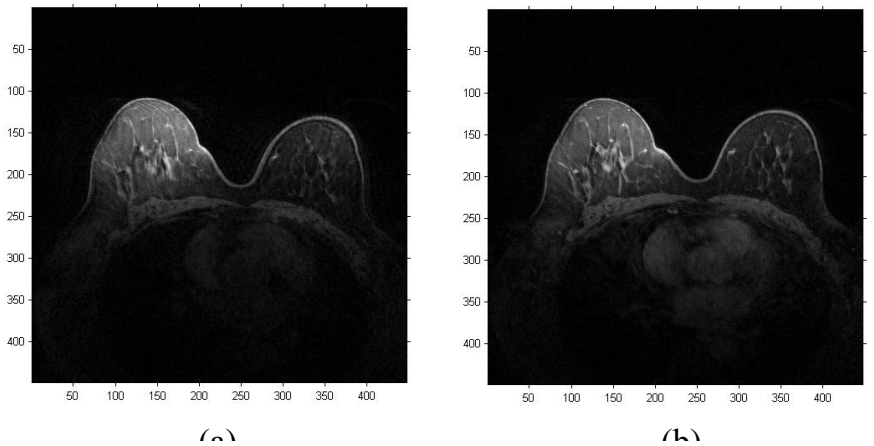

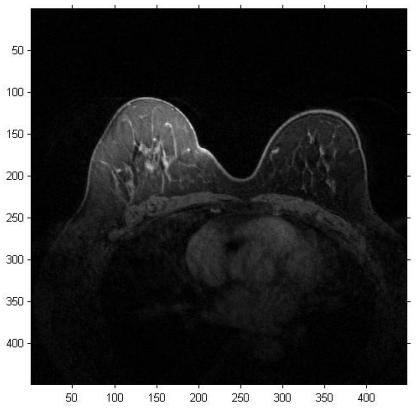

(c)

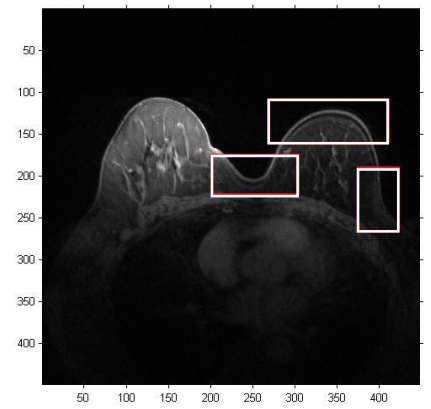

(d)
Figure 1 Original slice at (a) first minute, (b) second minute, (c) third minute and (d) the motion artefact (the white bounding boxes indicated the breast tissues are not in phase).

Background subtraction is a technique to indentify the moving objects from the portion of sequence slices that differs notably from a background model [7]. In order to increase the system efficiency, the simple technique as slice differencing and adaptive median filtering are more preferable. The slice difference is denoted by Equation (1):

$$
\mid \text { slice }_{\mathrm{i}}-\text { slice }_{\mathrm{i}-1} \mid>T h,
$$

where slice $_{\mathrm{i}}$ denotes the current slice, slice $_{\mathrm{i}-1}$ represents the background model and $T h$ is a user default thresholding value. By treating the pre-processing image as background model, the motion artefact in the images is detected and successfully removed from the slices.

Next, to improve the signal to noise ratio and enhance the system performance, the adaptive median filtering is employed. The median filter is a nonlinear digital filtering technique which often applied to eliminate the unwanted salt \& pepper noise. Figure 2 shows the result images after slice differencing and median filtering.

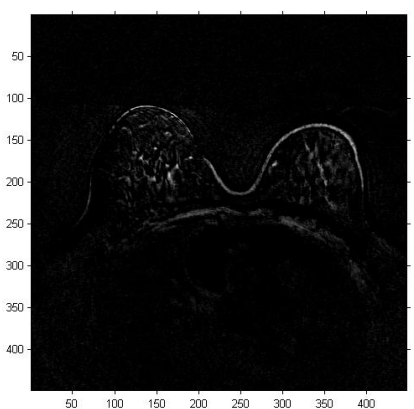

(a)

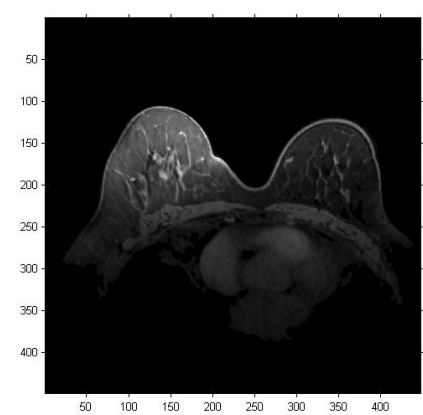

(b)
Figure 2 Slice differencing and noise elimination. (a) Motion artefact should be eliminated and (b) Image with removed motion artefact and filtered noise. 


\section{Horizontal Projection Segmentation}

In order to reduce the computational cost, the projection segmentation technique is employed to eliminate all the insignificant pixels except the breast, for instance, thorax and lungs regions. Firstly, the horizontal projection is exploited in order to eliminate abundance of insignificant contents. The horizontal projection $h(y)$ is the histogram obtained by counting the non-black pixels in each horizontal breast MRI scan. After that, a histogram based of row position $y$ is plotted accordingly.

Equations (2) - (4) define the horizontal lower and upper breast boundaries.

$$
\begin{aligned}
& y_{\text {min }}=y_{1}, \text { where } h\left(\min \left(y_{1}\right)\right)>0, \\
& k=k+1, \text { where } h\left(y_{2}\right)-h\left(y_{2}-1\right)>T r, \\
& y_{\max }=y_{2}, \text { where } k=2,
\end{aligned}
$$

where $h(y)$ denotes the horizontal projection histogram, $y_{\min }$ is horizontal lower boundary, $y_{\max }$ is upper breast boundaries, $k$ is counter, and $y_{1} \& y_{2}$ represent the row position of the MR image that from $1,2,3, \ldots, 448$. Figure 3 shows the segmented breast by horizontal projection.

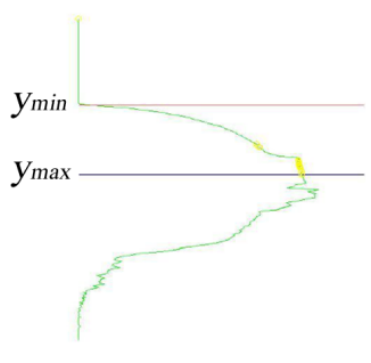

(a)

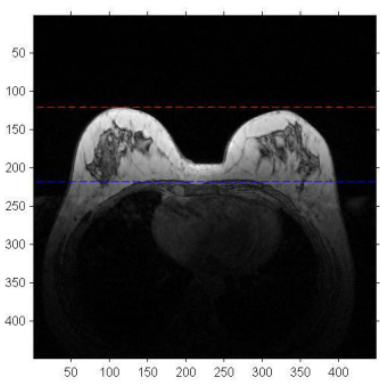

(b)
Figure 3 Horizontal projection breast segmentation. (a) Horizontal projection histogram, and (b) Segmented breast section.

The number of yellow circle sequence in Figure 3 indicates the number of counter $k$. From Figure 3, the proposed scheme is able to create a boundary which encloses the significant breast ROI. Then, the survived breast ROI are undergo vertical projection segmention.

\section{Vertical Projection Segmentation}

The vertical projection is employed to remove the hypodermic tissues that are near the sternum. Similiar to horizontal projection segmentation, the vertical projection $V(\mathrm{x})$ is the histogram obtained by counting the non-black pixels in each vertical scan at column position $\mathrm{x}$.

Equations (5) and (6) denote the hypodermic tissue boundary to be eliminated.

$$
\begin{aligned}
& X_{\text {min }}=x_{1}, \text { where } \mid V\left(x_{1}+i\right)-V\left(x_{1}+\left.(i-1)\right|_{i=0,1,2}>T r\right. \\
& X_{\text {max }}=x_{2}, \text { where } \mid V\left(x_{2}-(i-1)-\left.V\left(x_{2}-i\right)\right|_{i=0,1,2}>\operatorname{Tr},\right.
\end{aligned}
$$

where $V(x)$ represents the vertical projection histogram, $X_{\min }$ and $X_{\max }$ denote left and right boundaries for hypodermic tissues to be eliminated, and $x_{1} \& x_{2}$ correspond to the width of the MR image.

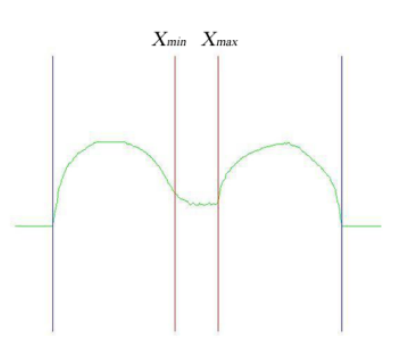

(a)

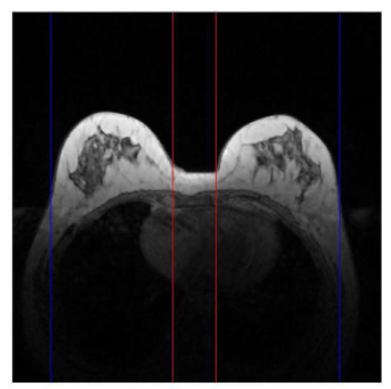

(b)
Figure 4 Vertical projection breast segmentation. (a) Horizontal projection histogram, (b) Segmented breast, and (c) Resulting image after horizontal and vertical projection.

Figure 4 demonstrates the result of segmented breast after vertical projection. From Figure 4(b), we can observe that the tissues between the breasts are eliminated. Much of the computational cost is saved due to only the segmented breast ROI will undergo image post-processing. Besides that, the thresholding is apply to the remained ROI. The darker pixels are convert to black colour as background while the brighter regions are convert to white colour as foreground.

\section{E. Edge Detection}

After thresholding, gradient filtering as equation (7) using the Sobel filter with matrix size $3 \times 3$ shown in Figure 5 is used to detect breast ROI edges. After the Sobel filtering, the breast ROI is totally segmented.

$$
\nabla f=\operatorname{grad}(f) \equiv\left[\begin{array}{l}
g_{x} \\
g_{y}
\end{array}\right]=\left[\begin{array}{l}
\frac{\partial f}{\partial x} \\
\frac{\partial f}{\partial y}
\end{array}\right],
$$

where $f$ is image gradient defined by two orthogonal components, $g_{x}$ and $g_{y}$.

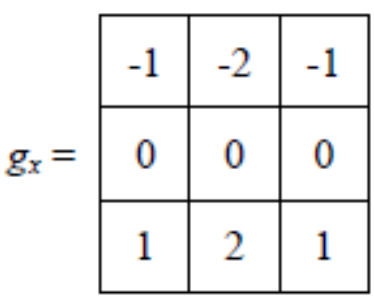

(a)

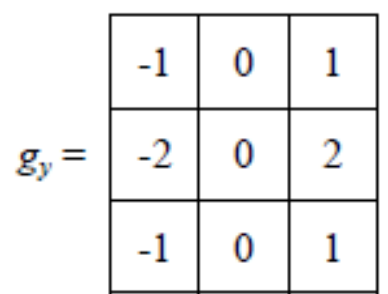

(b)
Figure 5 Sobel gradient matrices for edge detection in (a) vertical $\left(g_{x}\right)$ and (b) horizontal $\left(g_{y}\right)$ direction. 


\section{RESUlts}

Algorithm proposed in this paper is tested on 55 sets of MRI breast images. Totally 110 slices from 55 different patients ( 2 images from each patient) have been analyzed. Each image has resolution of $448 \times 448$ pixels with colour depth 16 bits per pixel. All images are stored in compliance with DICOM standard. In order to standardize the experimental procedure, the slices at position $80^{\text {th }}$ and $90^{\text {th }}$ of each patient are being observed. Figure 6 shows the results for certain patients.

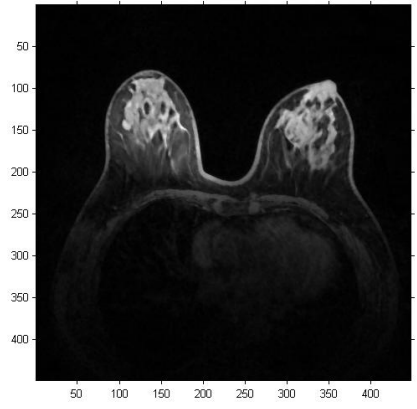

(a)

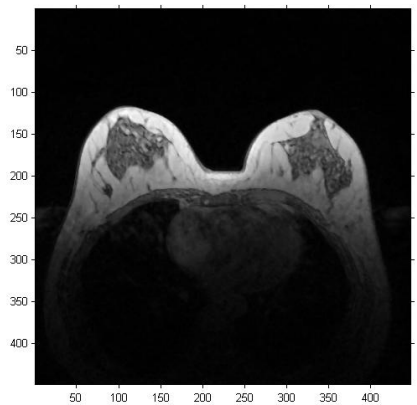

(c)

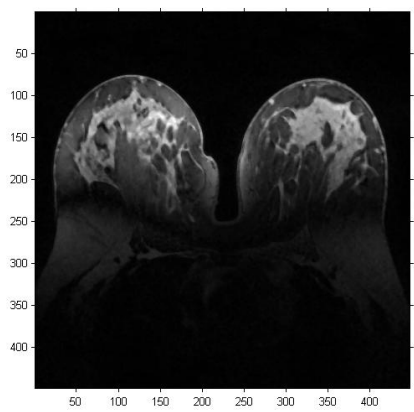

(e)

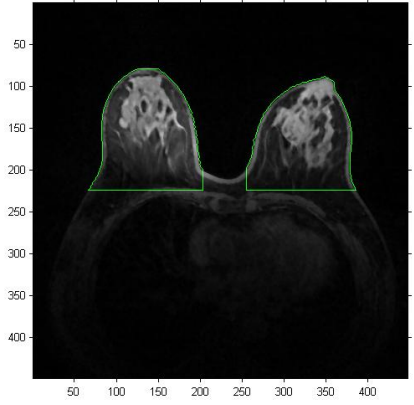

(b)

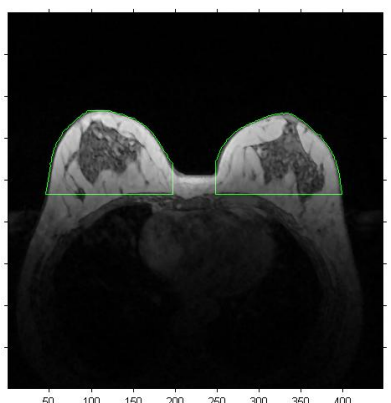

(d)

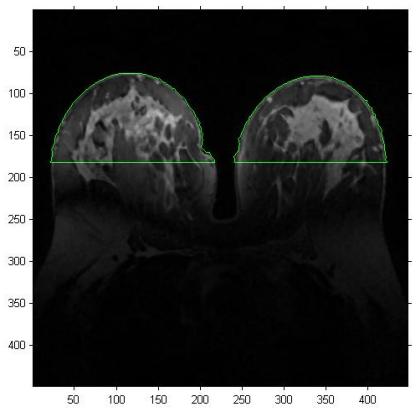

(f)
Figure 6 Segmented results. (a), (c) \& (e) are original DICOM images while (b), (d) \& (f) are the segmented result accordingly.

Results of breast ROI segmentation are divided into two groups which are "Acceptable" and "Unacceptable" as shown in Table 1. An "Acceptable" segmentation should boundary $85 \%$ of breast tissues, for instance, from the inner lining of milk ducts or the lobules to the nipples. Figure 6(a), 6(b), 6(c) \& 6(d) represent the "Acceptable" segmentations. On the other hand, if the segmented breast tissues by the proposed scheme are less than $85 \%$, the case is classified as "Unacceptable" segmentation. Figure 6(e) \& $6(f)$ denote the "Unacceptable" segmentations. Total percentage for "Acceptable" and "Unacceptable" results are shown at the end of each column.

\begin{tabular}{|l|c|c|}
\hline \multicolumn{2}{|c|}{ TABLE 1. Breast ROI segmentation results } \\
\hline Result & Acceptable & Unacceptable \\
\hline Total No.(110) & 102 & 8 \\
\hline Percentage & $92.72 \%$ & $7.28 \%$ \\
\hline
\end{tabular}

From the result showed in the Table 1 . The proposed method for detecting the breast border contour works well on our test set containing 110 breast MRI images. $92.72 \%$ breast ROI can be correctly segmented due to the main reason that well work done in pre-processing stage that provides the high quality images. On another perspective, $7.28 \%$ of breast ROI cannot be segmented in acceptable range due to the special case for the screening breast, for instance, fat saturate breast.

\section{CONCLUSION}

Based on preliminary testing, the procedure we have developed for identifying the ROI in breast MRI images works well and it is ready to assist hand-drawn ROIs for use in breast cancer research. However the improvements are still needed, although the proposed scheme works in most of the cases. More conditions may be added into vertical and horizontal projections segmentation, which could be segment the breast ROI more accurately and reliable. Besides, more of the different breast screening cases and information are also required to enlarge the segmentation database. The situations in which it fails are very minority, so it would appear that the proposed approach is a capable and high efficiency system.

\section{ACKNOWLEDGMENT}

The breast MR images used in this research are obtained from Malacca General Hospital, Malaysia.

[1] American Cancer $\begin{gathered}\text { REFERENCES } \\ \text { Society, Internet side address }\end{gathered}$ http://www.cancer.org/Research/CancerFactsFigures/index

[2] R. M. Rangayyan, L. Shen, et al. Improvement of Sensitivity of Breast Cancer Diagnosis with Adaptive Neighbourhood Contrast Enhancement of Mammograms, " IEEE Trans Inf Technol Biomed. 1 (1997) 161-170.

[3] M. Kriege, C. T. M. Brekelmans, et al. Efficacy of MRI and Mammography for Breast-Cancer Screening in Women with a Familial or Genetic Predisposition, N Engl J Med. 351 (2004) 427437.

[4] C. D. Lehman, J. D. Blume, et al. Screening Women at High Risk for Breast Cancer with Mammography and Magnetic Resonance Imaging, Wiley InterScience. 103 (2005) 1898-1905.

[5] M. J. Stoutjesdijk, C. Boetes, et al. Magnetic Resonance Imaging and Mammography in Women With a Hereditary Risk of Breast Cancer, Journal of the National Cancer Institute. 93 (2001) 10951102 .

[6] B. B. Chaudhuri and U. Pal. A complete printed bangla ocr system. Pattern Recognition, 31(5): (1998) 531-549.

[7] S. G. Moore, P. J. Shenoy, L. Fanucchi, J. W. Tumeh, C. R. Flowers, Cost-effectiveness of MRI compared to mammography for breast cancer screening in a high risk population, BMC Health Serv Res. 9 (2009) 9-10. 